\title{
Notes sur l'Exploitation du Cheptel cambodgien
}

\section{INCLINATION DES CAMBODGIENS POUR L'ELEVAGE}

par R. BARADAT

ON s'accorde à trouver chez le Cambodgien des qualités de bouvier que ne possède pas l'Annamite. Fruste et observateur, le paysan du Cambodge sait ménager son bétail, réduire la durée des attelées à la cadence souhaitable pour la rumination, cette modération dans l'effort étant partagée aussi par le bouvier. Les soins ordinaires (abreuvement, bains, distribution de nourriture) sont régulièrement dispensés. Cette sollicitude ne s'étend guère qu'aux animaux de trait, tandis que le troupeau d'élevage est négligé. Le bétail se trouve le plus souvent parqué, sans toit, sans litière, à même la terre nue que les pluies et les déjections transforment en cloaque. Peu soucieux de confort pour eux-mêmes, les Cambodgiens ne songent guère à fournir un abri à leur bétail. Ils ne pensent pas davantage à constituer des réserves fourragères en prévision des disettes saisonnières.

La diététique est basée essentiellement sur le pâturage, la nature étant chargée de l'entretien des animaux. L'homme n'intervient guère que pour l'exploitation des facultés motrices. On connait les qualités d'observation que l'on trouve chez les éleveurs cambodgiens. Ces conceptions zootechniques sont basées sur la tradition et, pour autant, sur des survivances superstitieuses. Elles ne traduisent aucunement une science de l'élevage. Il n'existe aucun souci de sélection dans le choix des taureaux à réserver pour la reproduction.

En bref, le comportement du bouvier montre des vertus négatives plus qu'il ne marque l'assiduité, l'attachement et le savoir.

C'est dans le même sens qu'il convient d'interpréter la conduite traditionnelle inspirée de l'enseignement bouddhique. La fidélité aux préceptes (Sappa pasak akkaranam : Tu ne maltraiteras pas les animaux - Pana tekpa tor : Tu n'ôteras pas la vie à l'être qui la possède) se traduit par le souci de ne pas maltraiter les animaux, mais n'implique pas un effort d'assistance. La répugnance manifeste pour tout ce qui touche aux sacrifices d'animaux (Mangsak veak nekchéa : Tu n'élèveras pas les animaux pour qu' on fasse commerce de leur viande) tient le Cambodgien à l'écart du commerce de boucherie dont se chargent les Musulmans (Chams, Afghans et les Chinois). Ce conformisme religieux est, peut-être, un frein à l'essor de l'élevage; il est obstacle notamment à toutc ocuvre de sélection. Le bétail n'étant exploité que pour ses qualités motrices, l'engraissement, la production de la viande sont considérés comme secondaires.

Aussi, le Cambodgien n'est-il guère porté à tirer parti des immenses parcours sur savane boisée où la densité du bétail pourrait être augmentée. On n'y rencontre pas de populations pastorales vivant du revenu de leurs troupeaux comme il est de règle en Afrique dans des conditions mésologiques semblables. Rares sont les troupeaux importants. Il y a quelques annécs, on ne put dénicher un seul Cambodgien possédant un élevage suffisant pour mériter d'être inscrit au Groupement professionnel des Eleveurs, les seuls inscrits pouvant ètre trouvés parmi les éleveurs français et afghans. Il faut dire que la possession d'un fort troupeau est un signe apparent de richesse et, dans les villages reculés, un prétexte à l'attention intéressée du mandarin. Pour si exceptionnelles qu'elles soient, les malversations contrariènt l'essor de l'élevage, s'opposent à un surcroît de production et font obstacle à l'enrichissement génćral.

L'augmentation numérique du cheptel est gravement menacée par les vols d'animaux. Dans les territoires proches des frontieres où il est facile de dissimuler les animaux volés, de dérouter les recherches, les razzias de bétail prennent parfois l'allure d'entreprises étendues, englobant de nombreux complices, sous la protection occulte des autorités communales. En matière d'élevage, les progrès sont lents, les pouvoirs publics auront donc tout loisir pour asseoir parmi les fonctionnaires ruraux le goût des travaux rustiques, la sollicitude envers les entreprises paysannes et le désintéressement.

Les Chinois, qui sont un stimulant efficace dans bien des compartinents de l'économie du pays; jouent un rôle effacé en matiere d'élevage, sauf dans les élevages de basse-cour (porcins et volailles) où leur activité est prépondérante pour le placement des jeunes sujets et le commerce des adultes. Tradjtionnellement, le Chinois n'est pas porté vers l'élevage des bovidés dont le rendement lui apparaît, au surplus, aléatoire et à échéance trop lointaine. Il apparaît nécessaire, cependant, de stimuler les Cambodgiens qui ont accoutumé de ne pas porter leurs efforts au delà de leurs besoins immédiats. Pour les motifs que je viens d'énumerer les 
mandarins et les petits fonctionnaires d'autorité sont mal préparés à cette tâché. Par leur intermédiaire, l'intervention des pouvoürs publics, en matière d'élevage, demeure généralement inefficace. On devra arriver, par paliers; à urie intervention directe du Crédit populaire agricole soús le mode de mutuelles d'élevage inspirées des Sociétés de prévoyance d'A.O.F.

I.'exploitation des ressources náturélles - forêts, agriculture, élevage - n'est pas; au. Cambodge, doublée d'industries de transformation. Le Cambodgien reste un rural, pénétré du sens de la nature et peu incliné vers l'ịndustrie manüfacturière. La force et la stabilité de ge type humain résident dans l'attachement au sol et le maintien de la tradition. Avec une natalité faible, menácé pair de's voisins plus entreprenants, le Cambodge ne pourrä affirmer sa force de résistance que si elle est consolidée par une politique de défense des intérêts paysans, visant à une amélioration de l'agriculture ét de l'élevage.

\section{FÉCONDITÉ DES FEMELIES ET CADENCE DES MISES BÄS}

La fécondité viable des vaches et bufflessés resso'rt bien médiocre des statistiquès. Pour l'annèe 1945, elle serait de $41,3 \%$ pour l'ensemblè du troupeau bovin avec un maximum de $62,7 \%$ à KompongChnang et un minimum de $25,4 \%$ à Siem-Réap. Elle est dé $30,9 \%$ pour l'ensemble des'bufflesses avec maximum de $49,8 \%$ à Svay-Rieng contre $22,5 \%$ à Siem-Réap. Une certaine concordance d'une province à l'autre permet de ne pas rejeter ces chiffres.

La fécondité viable résulte de la natalité diminuée de la mortalité durant lca trois premières années d'âge. Récemment, au cours de multiples enquêtes, j'ai essayé d'approcher les chiffres de fécondité réelle.

Je citerai ceux rélevés à l'occasion des concours itinérants successifs dans la province de KompongSpeu (enquête portant sur 1.500 vaches environ et dans les mêmes villages).

$$
\left.\begin{array}{l}
1941: 69,57 \% \\
1942: 76,11 \% \\
1943: 71,08 \%
\end{array}\right\} \text { Moyennè de fécondité sur }
$$

En 1942 , la fécondité varie de $88,88 \%$ (maximum) à 65,06 (minimum), chacun de cés chiffres se rapportant à l'ensemble du troupeau d'un village.

Sur les bufflesses, la moyenne relevée est de $61,70 \%$ avec maximum de $72 \%$ et minimum de $43 \%$ selon les villages.

Les variations de fécondité, d'un village à l'autre, tiennent à la valeur des pâturages ou à la faveur des précipitations atmosphériques qui accélerent lá pousse de l'herbe à l'époque du rut. Chose digne de remarque, chez les bovidés et cervidés sauvages, la saison du rut est conditionnée tant par la présence de vitamine $\mathrm{E}$, phosphore et protéine' dans l'herbe nouvelle que par la durée de la gestation, les espèces à gestation de, longue durée entrant en rut les premières. 'Comme si. par une attention particulière, la nature disposait les futures mises bas à l'époque où les jeunes produits seront à l'abri des météores fâcheux. Sur les bovidés domestiques, entretenus souvent dans des' conditions, proches de l'état naturel, la saison du rut reste marquée par une accentuation des phénomènes dụ cycle cestral, moins perceptibles durant le reste de l'année.

La maturité sexuelle chez la vache apparaît entre deux ans et demi et trois ans, selon les régions, Il ne semble pas qu'un grand nombre de saillies soit nécessaire chez la génisse. La plupart des génisses se trouvent fécondées au moment où apparaissent les deuxièmes mitoyennes, si bien que la'moyenne des premières mises bas s'établit vers quatre ans:

Le retour des chaleurs s'observe génëralement vers la fin du deuxième mois qui suit le vêlagè Il est toutefois beaucoup plus tardif quand la vache nourrit péniblement son produit en saison de disette fourragère. La mort du produit, l'arrêt de la sécrétion lactée hâtent le retour du cycle cestral, même en saison où les pâtures sont maigres et desséchées.

La proportion des saillies à résultăt positif est beaucoup plus grande de février à mại, qui est la saison de repousse de l'herbe après les feux de brousse, ou sur les rizières en chaume abreuvées des premières pluies. C'est aussi la saison où l'cestrus est sinon plus durable, du moins plus apparent, et sollicite fortement les taurillons nombreux, en général, dans le troupeau. On doit noter que beaucoup de bouufs castrés par martelage conservent un instinct scxuel amoindri qui, dans l'opinion des Cambodgiens, leur donne une capacite de géniteurs: La castration, telle que la pratiquent les Cambodgiens, reste souvent incomplète, laissant persister. des îlots de tissu interstitiel, si bien que certains caractères sexuels sont conservés grâce a la sécrétion hormonale. D'où cette croyance, que l'on peut retrouver chez la plupart des eleveurs", 'qu'il est inutile de réserver un taureau puisquela la procréation peut être assurée par les bceufs. Beaucoup de ces eunuques ne sont pas, en effet, suffisamment neutralisés, l'excitation géméralé n'est pas totalement supprimée et on peut les voir harceler les femelles en état d'appétit génésique. Il reste difficile de faire admellre aux Cambodgiens que la fécondation des femelles ne vient pas des boufs, mais des taurillons nombreux dans le troupeau.

La plus grande proportion des mises bas sur savane boiséé, dans des conditions d'entretien semi-naturelles, s'observe dè novembre à février. Nous avons relevé les pourcentages suivants' : novembre $14 \%$, décembre $21 \%$ ' janvier $29 \%$, février $10 \%$. mars $5 \%$. Autres mois $21 \%$. 
L'influence des conditions mésologiques peut être ainsi définie : sur terres opulentes des berges, la précocité est plus grande, l'âge de la première mise bas étant fréquemment 3 ans. Le maximum des naissances est observé en novembre. La fécondité moyenne dépasse $83 \%$.

Sur parcours chiches des terrains sablonneux, où la disette fourragère est intense en saison sèche, la fécondité moyenne est de $69,3 \%$ et le maximum des naissances observé de mars à mai. Il faut voir là, au travers de la domestication, une action de la nature pour guider les naissances jusqu'à l'époque où, avec une nourriture abondante et aqueuse, les vaches pourront produire le lait nécessaire au jeune produit.

Avec des soins réguliers, l'entretien partiel à l'étable, la distribution d'aliments concentrés, la périodicité dı cycle œestral s'affranchit des conditions naturelles. Ainsi en 1944, sur le troupeau de la plantation de Thmâr-Pit, on observe $72,4 \%$ de naissances reparties comme suit : novembre à janvier $41 \%$, février à avril $18 \%$, mai à juillet $21 \%$, août à octobre $20 \%$. Durant le même temps, on observait à la plantation de Chup, sur un effectif de 262 vaches, une proportion de naissances de $80,8 \%$. Mais, quelques femelles provenant d'achats récents, l'examen de la cadence des mises bas serait moins probant.

De ces observations sur la cadence des mises bas, on pourrait conclure que le début de la saison sèche (décembre à janvier) constitue la saison la plus favorable aux naissances, car les jeunes produits se trouvent plus âgés et agucris pour affronter les pluies de la mousson. Une manifestation de ce rythme se retrouve sur les pâturages opulents des berges, où il est meme accentué, et en savane boisée, ou les parcours illimités mettent le bétail à l'abri des rigueurs de la saison sèche. Par contre, dans les contrées rizicoles à forte densité de bétail, où les investigations alimentaires sont restreintes, la fonction mammaire est compromise par l'âpreté de la saison sèche; aussi la fréquence des mises bas devient-elle plus forte en arrière-saison.

Cette adaptation s'est faite sans l'intervention de l'homme. Elle est d'ailleurs précaire, car dans les élevages bien conduits où les animaux sont abrités des influences saisonnières par une amélioration de leur diététique, les mises bas tendent à s'échelonner en toutes saisons.

On pourrait trouver souhaitable une intervention de l'homme pour guider les fécondations et faire aboutir les naissances à l'époque la plus favorable. Cette pratique suppose la mise à part des taureaux et taurillons, leur séjour dans le troupeau étant limité à la saison que l'on juge propice. Une telle intervention est possible dans un élevage très surveillé, à la condition de posséder des taureaux en nombre suffisant et de soutenir leur ardeur génésique par une alimentation appropriée. Elle demande cependant quelques précautions en raison de la brieveté des chaleurs et de l'augmentation du nombre des saillies nécessaires pour assurer la fécondation en saison des pluies. Elle suppose, d'autre part, l'attribution d'un complément de ration aux femelles pour favoriser l'activité ovarienne. Cette discipline de la fécondation ne peut être retenue dans la condition attardée de l'élevage au Cambodge. Mal conduite, elle ne pourrait qu'abaisser le taux de fécondité. Mieux vaudra s'attacher à la protection des jeunes en bas âge, qui parait plus aisément réalisable.

L'avortement est relativement rare, en moyenne inférieur à $1 / 100$ des gestations. On observe cependant de veritables bouffées d'avortements sporadiques, la maladie de Bang étant inconnue. Nous avons ainsi relevé dans un même troupeau 24 avortements sur 153 gestantes, soit $15,7 \%$, le retentissement étant nul sur l'organisme maternel, puisquc, l'annćc suivante, la fécondité redevenait régulière : $71,48 \%$. Est-ce là une marque d'avitaminose à là suite d'une sécheresse prolongée? Je ne le puis affirmer.

De la série des enquêtes que j'ai effectuées, je me permets de conclure que la fécondité moyenne, sur l'ensemble des vaches du Cambodge, doit être évaluée à $72 \%$, moyenne tout à fait honorable.

Sur la bufflesse, la maturité sexuelle' apparait en général vers 3 ans, la gestation dure un bon mois de plus que chez la vache (onze mois). L'âge moyen de la première misc bas varie de quatre ans et demi à cinq ans selon les régions. Après mise bas, les chaleurs réapparaissent un peu plus tardivement que chez la vache (65 jours environ), mais la fécondation n'est généralement assurée que lorsque le produit est complètement sevré, la lactation cessant vers le $6^{e}$ mois. Il n'y a pas, entre vache et bufflesse, de différence sensible dans le retour du cycle cestral ( 3 semaines chez la vache et 4 semaines chez la bufflesse). Elle ne peut servir à justifier l'abaissement du taux moyen de fécondité qui, de $72 \%$ chez la vache, est seulement de $61 \%$ chez la bufflesse.

Mais les mâles non castrés sont, dans le troupeau bubalin, en nombre parfois insuffisant pour pouvoir rencontrer, à temps, toutes les femelles dont les chaleurs sont, de surcroît, assez curieuses.

L'œstrus dure trois ou quatre jours, 'les femelles témoignent fortement; mais, soit pruderie particulière à cette espèce, soit action inhibitrice de la chaleur solaire, il y a fort peu de saillies au pâturage. La rencontre des sexes se fait le soir, au retour du pâturage, ou dans l'enclos de parcage nocturne. La timidité des jeunes bufflons les maintient longtemps dans une indifférence génitale, alors que les taurillons interviennent de façon plus précoce et jouent un rôle actif dans la reproduction. 
La durée des chaleurs, chez les vaches et bufflesses, est heureusement influencée par la repousse de l'herbe aux premières pluies qui met fin au régime déficitaire de saison sèche. Dès que l'herbage s'enrichit en vitamines, phosphore, éléments azotés, la proportion augmente des saillies fécondantes.

Les gestations gémellaires, exceptionnelles dans les deux espèces, sont toutefois moins rares'avec la bufflesse.

\section{MORTALITÉ DES JEUNES}

La parfaite adaptation du betáil cambodgien au milieu, sa résistance face à des conditions alimentaires précaires contribuent à la réussite de la fécondation, qui se maintient selon un pourcentage que l'on a le droit de juger favorable.

Par contre, l'entretien en pâture dan's des conditions naturelles est un obstacle au développement des jeunes produits. Ceux-ci reçoivent une ration de lait réduile, sont sevrés très tôt, sunt atteints de précarences minérales, azotées, vitaminiques, de troubles digestifs, sont moins résistants aux infections parasitaires. Sur sols sablonneux, pauvres en humus, sur terres « battantes » des rizières dénudées par la sécheresse, les jeunes nés au début de la saison sèche sont presque condamnés à périr. Par auto-défense de l'espèce, le maximum des naissances y est observé assez tard en saison, sans cependant que les jeunes en bas âge soient à l'abri des irrégularités climatiques (sécheresse prolongée par exemple). Sur parcours de savane boisée, les mères et élèves supportent mieux les méfaits de la sécheresse grâce à l'étendue des investigations alimentaires. Mais le troupeau trouve ici un autre danger, celui des attaques des grands félins. La concentration du bétail autour des points d'eau, les allées et venues dans les galeries boisées qui bordent les cours d'eau facilitent l'embuscade des fauves. Les attaques sont dues aux diverses panthères (Khla angkruk, Khla dambang, Khla takhen, Felis Diardi, Félis pardus) beaucoup plus qu'au tigre.

Les veaux du premier âge, de petite taille (10 kilos environ à la naissance), sont sans aucune défense et une proie facile. Aussi, dans certains villages, sontils, durant le jour, enfermés dans le paro à bétail, proche des cases, où ils attendent pour téter que la mère rentre le soir du pacage. Ils ne sont agrégés au troupeau qu'à l'âge de 2 ou 3 mois, où ils sont capables de brouter.

Les rapts commis par les félins sont; dans certaines régions, très graves. Nous avons relevé, dans des villages, jusqu'à $32,5 \%$ des veaux et $41 \%$ des bufflotins on bas âge.

Les fauves sont un obstacle à l'intensification de l'élevage dans des régions qui' se prêteraient à la multiplication du cheptel, tandis que les éleveurs ne se soucient guère de se défendre des dommages causés à leurs troupeaux ou d'en accroitre les. revenus.

Les animaux nés plus tard en saison des pluies sont exposés aux refroidissements brusques des tornades, aux ondées sévères de la mousson de suroît. Ils sont encore trop chétifs et n'ont pas accumulé suffisamment de réserves pour aborder la sécheresse suivante.

Le déchet en bas âge par suite des précarences, des désordes digestifs, de l'inanition, est assez élevé. Nous avons observé jusqu'à $38 \%$ sur les veaux et jusqu'à $49 \%$ sur les buffletins, dans certains villages fortement éprouvés.

Sur les produits de l'année, la mortalité pour l'ensemble du Cambodge est très élevée; de l'ordre. de $25 \%$ en moyenne, la cadence des pertes pouvant être déterminée comme suit : avril à mai : $7 \%$ (méfaits' de la saison sèche), août à octobre : $10 \%$ (saison des grandes pluies el lornades), autres mois : $8 \%$.

Le taux de mortalité s'abaisse aux alentours de $5 \%$ durant la deuxième année et diminue encore durant la troisième année.

Ces mêmes pourcentages de mortalité peuvent être retenus pour les jeunes bufflons. La sécheresse ne laisse pas de leur être sévère, bien que cet élevage soit réparti dans des régions basses, marécageuses ou dans des cantons boisés où il est parfois le seul possible en raison de la fréquence des agressions. de fauves. Quoique les bufflesses fassent front contre les attaques des grands félins et défendent courageusement leurs petits, le chiffre des victimes reste élevé.

Même dans des conditions d'entretien favorables (étable, abri, aliments concentrés), la mortalité sur les jeunes reste élevée. Ainsi, à la plantation de Chup, sur 210 naissances en 1944 (109 veaux, 101 vêles), on relève 13 morts, soit $6,2 \%$. Tant il est vrai qu'une ration de lait déficiente, un sevrage hâtif ne peuvent être qu'une mauvaise préparation aux conditions sévères d'existence.

Le pourcentage de mortalité n'est pas, il s'en faut, aussi catastrophique dans tous les élevages du Cambodge. On le retrouve, souvent, inférieur au taux de mortalité de $10 \%$ admis par les divers auteurs pour les élevages tropicaux. Néanmoins, les ravages des. diverses enzooties, l'imprévoyance des éleveurs sont tels que j'estime à $25 \%$ la mortalité en bas âge sur l'ensemble du cheptel cambodgien.

Les effectifs du jeune bétail portés sur les recensements sont inférieurs à la réalité. Au cours de divers sondages, il a été observć, dans: les villages, que le nombre des produits âgés de moins de 2 ans était largement supérieur à celui des femelles adultes pour les vaches et légèrement inférieur pour 
celui des bufflesses. Dans les régions rizicoles à forte densité de bétail, les jeunes produits forment $60 \%$ du troupeau d'élevage, les vaches $40 \%$. Sur terre de berge, où leur sauvegarde est mieux assurée, les jeunes produits représentent $65 \%$ du troupeau d'élevage. Dans le premier cas, la fécondité viable égale $50 \%$; dans le second, elle est de $60 \%$, et un nombre de vaches inférieur de $17 \%$ suffit à assurer la même production.

L'inattention pour les produits du premier âge, dont l'existence est précaire, incline les autorités communales à négliger cette production dans l'évaluation des effectifs. Les recensements ne concernent guère que les sujets ayant franchi le cap du sevrage total. D'autre part, l'indifférence pour les produits femelles les fait négliger.

\section{CHARGE ET ENCOMBREMENT DU PATURAGE}

Si l'on fait abstraction des terres opulentes des berges, où les conditions d'entretien du bétail sont particulierement favorables, les possibilités d'accroissement du cheptel paraissent atteintes dans la.plupart des contrées rizicoles. Ce serait d'un optimisme injustifié que d'y vouloir encore surcharger le pâturage (Takéo 33 têtes, Svay-Rieng 31 têtes au kilomètre carré), alors que la sécheresse dépouille les rizières après la récolte et y laisse subsister de pauvres chaumes où le bétail s'attarde. les préférant aux herbes ligneuses de ses parcours habituels. Quoique superficiels, les labours de la rizière améliorent les qualités physiques du sol et modifient heureusement la composition du pâturage et sa capacité de charge. Ils permettent aux fines graminées annuelles de n'être pas étouffées par les espèces grossières et vivaces. La transformation est telle que la composition du pâturage sur les vieilles rizières est totalement différente de celle sur savanes incultes.

Il est bien difficile d'évaluer le nombre de têtes de bétail qu'un hectare peut porter. Pour les races autochtones, rustiques, et sur savane tropicale, les auteurs s'accordent sur le chiffre de une tête pour autant d'hectares qu'il y a de mois de sécheresse. Au Cambodge, à condition que les points d'eau y soient d'accès facile, il serait possible d'entretenir une quinzaine de bovidés sur un kilomètre carré de forêt claire grâce aux seules ressources naturelles de la vaine pâture.

D'immenses parcours offrent ainsi des possibilités d'élevage extensif (provinces de Kompong-Speu, Kampot, Pursat, Kompong-Chhnang, Kompong-Thom et Kratie). Il y faut seulement une politique de peuplement humain qui doit aller de pair avec l'extension de l'élevage (protection contre le paludisme, la piraterie) et un équipement rural sommaire relevant surtout de l'hydraulique pastorale. Nul doute que le cheptel de ces provinces pourrait ètre doublé en moins de dix ans. Sur ces savanes boisées, vit actuellement un cheptel sauvage d'environ 30.000 têtes que Ch. Dumas évalue comme suit : 20.000 banteng, 2.000 bcufs gris, 4.000 buffles sauvages et 5.000 gaurs (Ch. Dumas - La faune sauvage du Cambodge, p. 15). Ces mêmes territoires pourraient porter, après quelques aménagements, un cheptel de 300.000 boeufs et buffles domestiques.

Mais le gain de terres nouvelles ne peut permettre un accroissement illimité du cheptel. L'élevage du Cambodge n'est pas encore orienté vers une restauration de la fertilité du sol. Beaucoup de parcours sont maigres, épuisés par une raréfaction progressive de l'humus, fatigués par l'incendie annuel. On peut tenir pour presque nulle l'action des déjections animales à l'occasion de la vaine pâture, si même le surpeuplement et lé piétinement ne sont, par endroits, une cause de dégradation.

Le mode même d élevage s'oppose à un accroissement continu, celui-ci ne pouvant résulter que d'une transformalion progressive des méthodes en usage. Pour montrer les dangers qui guettent l'élevage cambodgien à une échéance moins lointaine qu'on serait porté à le croire, je rappellerai le grief d'érosion du sol et du pâturage que les éleveurs avertis retiennent contre les troupeaux trop nombreux dans l'Inde et au Kenya. Des milliers de bestiaux sans aucune valeur drainent les ressources végétales, ne rapportant presque rien jusqu'à leur mort qui survient par inanition, épuisement, pour le seul profit d'une peau de mauvaise qualité. Ceci' a amené les autorités britanniques à favoriser le décongestionnement des troupeaux. Dans certaines régions du Carmbodge, une propagande devra s'exercer pour montrer aux paysans qu'il est absurde de posséder un nombre de têtes supérieur à celui que peut nourrir le sol, qu'il est indispensable de se séparer du bétail inutile, qui mange autant que l'autre, et qu'on doit s'efforcer de 'sélectionner et conserver les sujets d'un meilleur rendement.

Avec les réserves d'usage devant la traitrise des précisions chiffrées, je risquerai une évaluation de la capacité de charge des parcours ordinaires en différentes régions du Cambodge.

Terres de berge et d'arrière-berge : 100 à 150 bceufs (de 250 kilos l'un) par kilometre carré, de décembre à juin, avec période optimum en février et mars. De juillet à novembre, ces terres sont en presque totalité inondées.

Sols de rizières sur fond siliceux ou argilo-siliceux : 20 à 30 bœufs au kilomètre carré. Ces terres pourraient être améliorées par fumures répétées, mais il semble qu'il soit malaisé d'en doubler la capacité de charge. 
Sols de savanes bordant le massif des Cardamómes, généralement bien pourvus de points d'eau (Kompong-Speu, Kampot, Kompong-Chnang, Pursat) : 12 à 20 bœufs par kilomètre carré.

Sols de savanes plus arides en saison. (SiemRéap, Kompong-Thom, Kratié) : 8 à 12 boeufs au kilomètre carré. La composition de la flore herbacée est sensiblement identique sur les deux savanes, .mais l'éloignement des points d'eau y oblige le bétail à des fatigues inutiles.

Ces sols médiocres, arénisés en surface, dégradés par les feux de brousse, sont recouverts d'un peuplement espacé de diptérocarpées et d'un revêtement de graminées grossières et de bambou nain. La suppression des feux de brousse, en permettant la restauration du sol, augmentera la capacité de charge. Pour favoriser la repousse de l'herbe aux premières pluies, la dégager du feutrage de l'ancienne végétation, la rendre accessible au bétail, los autochtones font passer le feu. Ce brîlage annuel est, dans certains cás, un mal nécessaire, mais il ne peut être toléré que s'il reste étroitement circonscrit.

Dans les régions où les pâlurés sont surchargées, il ne suffit pas d'interdire les abatages de femelles. Il faut pouvoir conserver les reproductrices qui, logiquement, doivent céder le pas au bétail nécessaire aux cultures. Détournées des tueries rurales, les reproductrices doivent être repoussées jusque dans l'arrière-pays, dans les régions de forêts claires où les possibilités spatiales sont considérables et les ressources fourragères incomplètement utilisées. Il existe un afflux de bétail de trait de l'arrièrepays vers les régions rizicoles, un courant inverse devrait emporter les jeunes femelles en excédent qui- encombrent les parcours limités. Ce serait la meilleure garantie de les soustraire à la tuerie. Un tel courant ne s'improvise pas, il est difficile à faire accepter d'éleveurs attardés dans leurs routines et peu enclins a suivre des suggestions. 'On y parviendrait cependant en prescrivant, pour toute famille paysanne habitant la haute région ou les vastes savanes, l'obligation, sous peine d'amende, d'entretenir un troupeau minimum de quatre vaches adultes ou de trois bufflesses.

Le dépistage des infractions à cette contrainte étant assuré par un agent subalterne du Khum dont la rémunération serait aisée par le produit des amendes. Cette fonction nouvelle pourrait porter le nom de " Chumtup péahanabal » ou agent auxiliaire chargé du bétail. Dans les régions reculées, où les déclarations d'épizooties sont tardives et incomplètes; le "Chumtup péahanabal » jouerait, de surcroît, un rôle analogue à celui qui est dévolu à l'auxiliaire du Service des Forêts; chargé de dépister et signaler les feux de brousse, le "Chumtup aranhabal ». Cet agent subalterne, désigné par les autorités communales, aurait une action majeure dans l'économie rurale.

Compte tenu du cheptel existant, des possibilités spatiales, de la flore agrostologique, il est facile de dresser un inventaire des villages où cette contrainte doit être appliquée. On y pourrait procéder par, étapes, cette mesure étant progressivement accentuée pour accroître la production dans les régions où la vocation du sol affirme la primauté de l'industrie pastorale.

\section{LE MILIEU INCLÉIMENT}

La succession rythmique de la saison sèche et de la saison humide provoque une série de ruptures d'équilibre préjudiciables aux organismes livrés aux aléas de la vaine pâture. Durant la période d'arrêt de la végétation, les animaux font de l'autophagie, mangent leurs graisses et leurs muscles pour ne les reformer que difficilement en saison d'abondance où leur ration est à peine normale.

TABLEAU I

Analyses de Sols - Embouche d'arrière-berge

\begin{tabular}{|c|c|c|c|c|c|c|c|}
\hline & KG-CHAM & KG-CHAM & KANDAL & PREY-VÈNG & TAKÉO & TAKÉO & TAKEO \\
\hline LIEU DE PRÉLĖVEMENT & $\begin{array}{l}\text { Arrière- } \\
\text { berge }\end{array}$ & $\begin{array}{l}\text { Arrière- } \\
\text { berge }\end{array}$ & $\begin{array}{c}\text { Arrrière- } \\
\text { berge }\end{array}$ & $\begin{array}{c}\text { Arrière- } \\
\text { berge }\end{array}$ & $\begin{array}{l}\text { Angkor- } \\
\text { Borey }\end{array}$ & $\begin{array}{c}\text { Angkor- } \\
\text { Borey }\end{array}$ & $\begin{array}{l}\text { Angkor- } \\
\text { Borey }\end{array}$ \\
\hline Nature et aspect de la végétation. & $\begin{array}{l}\text { Cynodon } \\
\text { Dactylon }\end{array}$ & $\begin{array}{l}\text { Cynodon } \\
\text { Dactylon }\end{array}$ & Para & Para & Para & $\begin{array}{l}\text { Cynodon } \\
\text { Dactylon }\end{array}$ & $\begin{array}{l}\text { Cynodon } \\
\text { Dactylon }\end{array}$ \\
\hline Azote total $0 / 00$ & 1,06 & 1,09 & 0,95 & '1,31 & 5,65 & 6,80 & 2,70 \\
\hline Chaux $0 / 00 \ldots \ldots \ldots \ldots \ldots \ldots$ & 5,98 & 2,17 & 4,66 & 3,41 & 2,96 & 2,50 & 1,75 \\
\hline $\begin{array}{l}\text { Acide phosphorique assimilable } \\
0 / 00 \ldots \ldots \ldots \ldots \ldots \ldots \ldots\end{array}$ & $-0,46$ & 0,05 & 0,90 & 0,39 & 0,26 & 0,20 & 0,05 \\
\hline Potasse assimilable $0 / 00$. & 0,15 & 0,10 & 0,22 & 0,17 & 0,25 & 0,46 & 0,16 \\
\hline p. $\mathrm{H}$ & 6,18 & 4,91 & 7,89 & 4,73 & 4,50 & 5,12 & 4,90 \\
\hline
\end{tabular}


Malgré l'adaptation du bétail indigène à des sols déficients, les carences phospho-calciques, l'hypovitaminose saisonnière diminuent la fécondité, contribuent à tarir les vaches en lactation, entravent la croissance des jeunes, diminuent la résistanco aux maladies infectieuses ou parasitaires. On voit apparaitre l'amaigrissement, l'anémie, la cachexie parasitaire.

L'inclémence du milieu retarde le développement des animaux, rabougrit le format, maintient la production et le rendement à un niveau médiocre. Elle s'oppose à toute introduction de races nouvelles et limite les possibilités d'amélioration par croisement.

On peut même imaginer le jour où, l'érosion du sol persistant (action du climat, feux de brousse, surcharge du pâturage), le cheptel, de plus en plus carencó, cesserait d'augmenter en nombre" pour rapidement dégénérer et baisser de format. La carence phospho-calcique ne parait pas jusqu'ici atteindre la fonction ovarienne au point de réduire la fćcondité globale. Néanmoins, elle intervient pour exagérer la mortalité dans le premier âge. Il est permis d'enregistrer dans cette manifestation comme un présage défavorable pour le devenir du cheptel cambodgien. A l'examen des récents travaux concernant les diverses carences des sols tropicaux et subtropicaux, on he peut manquer d'évoquer le danger de ce qui, pour le Cambodge, représente une "maladie d'avenir », beaucoup plus par les investigations qui devront être portées dans ce domaine que par une intensification rapide des troubles pathologiques ordinairement constates jusqu'ici.

Sur une moyenne de plusieurs années, les affections par carence atteignent plus gravement le potentiel de reconstitution du cheptel que les épizooties. C'est dire la nécessité d'y remédier. L'enrichissement en phosphore ou en chaux des pâtures, des sols cultivés parait hors de question pour des considérations économiques. Il faudra en arriver à instruire les éleveurs de la nécessité de distribuer aux animaux les éléments minéraux qui manquent dans le sol.

L'application d'un programme de-défense contre les carences minérales ne peut d'ailleurs être improvisée. Elle devra s'appuyer sur une série de recherches portant sur la composition des pâturages, l'analyse des fourrages et celle des sols. iVoir tableaux présentant schématiquement les analyses de quelques sols à vocation pastorale.) Ces analyses donnent une indication sur la vocation naturelle des pâtures, le fourrage des sols acides étant moins propre à l'élevage du bœuf.

TABLEAU II

Analyses de Sols - Savanes

\begin{tabular}{|c|c|c|c|c|c|c|c|}
\hline LIEU DE PRÉLĖVEMENT & SLAKOU & $\begin{array}{l}\text { BATTAM- } \\
\text { BANG }\end{array}$ & KAMPOT & $\underset{\text { BANG }}{\text { BATTAM- }}$ & PURSAT & PURSAT & PURSAT \\
\hline Élevage dominant & Bœufs & Bœufs & Bœufs & Buffles & Buffles & Buffles & Buffles \\
\hline Azote total $0 / 00$ & 0,78 & 0,81 & 0,87 & 1,15 & 1,09 & 1,32 & 1,90 \\
\hline Chaux $0 / 00 \ldots \ldots \ldots \ldots$ & 0,96 & 1,67 & 0,52 & 0,75 & 0,32 & 0,51 & 0,50 \\
\hline $\begin{array}{r}\text { Acide phosphorique assimilable } \\
0 / 00 \ldots \ldots \ldots \ldots \ldots \ldots \ldots\end{array}$ & 0,05 & 0,07 & 0,03 & 0,05 & 0,09 & 0,06 & 0,30 \\
\hline Potasse assimilable $0 / 00$ & 0,02 & 0,25 & 0,09 & 0,08 & 0,10 & 0,19 & 0,09 \\
\hline p.H... & & 5,16 & 5,25 & 3,70 & 3,94 & 4,82 & 4,10 \\
\hline
\end{tabular}

\section{LA CONTAGION}

Les méfaits des affections parasitaires du bétail sont considérables. Les parasitismes évoluent le plus souvent de façon sournoise, mais prennent aussi figure de maladies d'effectif. Les dommages qu'ils causent aux jeunes animaux ont été englobés dans l'estimation de la mortalité dans les premiers âges. Je ne préciserai pas davantage l'obstacle qu'ils représentent pour l'accroissement du cheptel.

Les diverses contagions (peste bovine, pasteu- rellose, charbons bactérien et bactéridien...) causent des pertes, sinon plus nombreuses, du moins plus dramatiques. La peste bovine figure en tête des contagions qui déciment le gros bétail. On sait la différence de sensibilité des deux espèces à cette maladie, les buffles y étant plus sensibles, et le rapport de la mortalité à la morbidité atteignant chez eux 80 à $95 \%$ selon les enzooties. Ce qu'on a moins présent à l'esprit, c'est qu'une épizootie de peste bovine ruine pour longtemps les facultés de reconstitution du cheptel. Après le passage de la 
contagion, on établit le décompte des morts, mais ce bilan, composé trop souvent à la légère, ne traduit pas l'incidence particulière de la maladie sur les jeunes en période de croissance, sur les femelles gravides ou en lactation. Les possibilités de restauration du troupealı sont compromises. On a tendance à négliger la gravité de cette position fâcheuse pour ne songer qu'aux attelages disparus, à l'entrave des façons culturales et aux impôts qui rentreront inal.

La peste bovine doit être considérée comme endémique sur le territoire du Cambodge et plusieurs de ses manifestations peuvent même passer inaperçues. En raison de la facilité des communications terrestres avec les pays voisins, Siam et Laos, des possibilités de transmission et diffusion par les ruminants et les porcins sauvages, la disparition complète de cette contagion ne peut être attendue de sitôt. La vaccination par les pulpes órganiques permet d'en restreindre les dégâts et la progression des doses de vaccin antipestique utilisées au Cambodge marque l'amélioration dans la défense du cheptel.

$$
\begin{aligned}
& 1931 \text {........... } 39.000 \text { doses } \\
& 1935 \ldots . . . \ldots .68 .000^{\prime}- \\
& 1941 \ldots \ldots \ldots \ldots .6147 .000 \\
& 1942 \ldots \ldots \ldots \ldots .305 .000- \\
& 1943 \ldots \ldots \ldots \ldots .378 .000 \quad- \\
& 1944 \ldots \ldots \ldots \ldots .397 .000-
\end{aligned}
$$

Depuis plus de dix ans que la méthode a fait ses preuves, il convient de dire le défaut d'enthousiasme des populations autochtones pour les interventions vaccinales. Au delà de la méfiance et de la routine paysanne, il y faut voir un manque de compréhension que ne peuvent entamer les témoignages les plus éclatants. C'est là une attitude peu compatible avec les qualités d'observation que l'on accorde au Cambodgien. Déviation de l'esprit inquiétante, car elle laisse entendre que le Cambodge connaittra encore les ravages de la peste bovine alors que ses voisins sauront s'en sauvegarder. La communauté d'aćtion, la solidarité paysanne sont encore trop grossières pour que les règles sanitaires les plus sages et les plus simples soient appliquées et conservent quelque efficacité.

La protection vaccinale ne peut, à elle seule, réaliser le miracle. Il lui arrive, d'ailleurs, d'être demandée trop tard et ce sont tous les aléas d'une intervention tardive. Il lui arrive aussi, et.c'est un comble, de ne pas être étendue à l'ensemble du cheptel et de laisser subsister suffisamment de matériel inflammable pour que l'incendie ne puisse $s^{\prime}$ éteindre. Le résultat est que les enzooties s'éternisent, que le vétérinaire avec sa seringue continue d'apparaitre et trop tard et trop peu, que se multiplient les pertes de bestiaux, tandis que les dépenses de vaccin s'accumulent pour un rendement décevant.

Le principe est, en effet, de devancer la contagion pour ne pas. s'essouffler à la suivre. Sauf en milieu dont le cheptel est exclusivement bovin, on est très rapidement débordé par elle. Chaque fois que les buffles représentent $25 \%$ de l'effectif total', il est nécessaire d'intervenir rapidement, de vacciner $75 \%$ au moins du cheptel, dont la quasi totalité des buffles. La lutte antipestique ne supporte pas l'inachevé. Elle est méthodique ou bien elle est vouée à I'échec.

L'effet à rechercher du vaccin est une action de masse plutôt qu'une protection individuelle absolue. On retire à la contagion les animaux réceptifs sur lesquels elle pourrait survivre et, en définitive, il suffit de conférer une immunité valable poirr la durée de l'épizootie. La règle du jeu veut donc que la contagion ne s'éternise pas au delà de la durée de cette protection et tout doit être mis en ceuvre pour obtenir ce résultat.

L'équipe d'opérateurs qui, seringue en main, se porte rapidement de village à village, vaccinant quelques animaux de ci de là, en négligeant bien davantage, est non seulement ridicule, elle est un danger public. Elle n'a pas quitté les lieux que l'épizootie continue de plus belle, gagne successivement la plupart des animaux réceptifs d'où elle attaquera plus tard le bétail traité, à cette phase où la protection vaccinale commence à s'évanouir. De telles interventions sont encore trop fréquentes; cllos ne modifient guère le cours des épizooties et discréditent le vaccin.

Ce défaut de methode ne pèse pas sur les seuls vaccinateurs pressés d'abréger leur office et s'accommodant d'une action superficielle. Il incombe davantage aux autorités communales et aux paysans qui n'apportent aucune aide au travail ingrat d'immunisation. Une part de responsabilité doit être également cherchée dans des échelons plus élevés de l'autorité provinciale. Durant ma carrière de praticion rural, jo n'ai jamais rençontré des fonctionnaires cambodgiens venus spontanément encourager par leur présence la réussite des interventions vaccinales. Pour un pays où la condition paysanne est de règle, un tel détachement venant de l'élite mandarinale ne laisse pas de surprendre. Il faut espérer que l'évolution politique actuelle; leur accordant une responsabilité plus étendue, les fonctionnaires cambodgiens se révèleront aptes à une coopération effective avec le Service vétérinaire dont les effectifs squelettiques ont besoin 'd'une assistance momentané sur les points d'intervention. Perfectionnement vers lequel il faut tendre et que. permettent d'escompter les' légers progrès enregistrés dans cette voie depuis quelques années. 
J'insiste sur ce fait que le vaccin antipestique est un outil qui ne vaut que par la façon dont il est manié. Il ne suffira pas au Cambodge d'avoir un. Institut Pasteur producteur de vaccins vétérinaires pour que soient réglés, d'un coup, tous les problèmes de la mise en œuvre. Durant de longues années encore, la peste bovine restera redoutable pour le cheptel cambodgien, elle le sera d'autant plus que l'armature administrative sera moins solide et que ne pourront être aplanies les difficultés venant des déclarations tardives et des interventions désespérées, presque de règle en raison des distances et des difficultés de communication.

Au moment où les grandes hécatombes de bétail ont disparu depuis quelques années, il est excellent de faire le point et de préciser l'importance des dommages que les diverses contagions, freinées par les vaccinations, continuent de causer annuellement au cheptel cambodgien. On peut évaluer le chiffre moyen de mortalité à :

\begin{tabular}{|c|c|c|c|c|}
\hline \multirow{6}{*}{$\begin{array}{l}\text { Mâles adultes... } \\
\text { Femelles adultes } \\
\text { Jeunes produits } \\
\text { Total......... }\end{array}$} & \multicolumn{2}{|c|}{ BOVINS } & \multicolumn{2}{|c|}{ BUBALINS } \\
\hline & Coefficient & Mortalité & Coefficient & Mortalité \\
\hline & $1 \%$ & 4.000 & $4 \%$ & 5.000 \\
\hline & $2 \%$ & 6.000 & $5 \%$ & 6.000 \\
\hline & $3 \%$ & $\underline{14.000}$ & $7 \%$ & 10.000 \\
\hline & & 24.000 & & 21.000 \\
\hline
\end{tabular}

Sur ces chiffres, la peste bovine figure pour $75 \%$ du total. La mortalité totale, imputable, en dehors des maladies contagieuses, aux affections banales, aux accidents, à la sénilité, est de $2 \%$ environ pour les boeufs, $8 \%$ pour les vaches, $8 \%$ pour les buffles et $13 \%$ pour les bufflesses.

De telles évaluations ne peuvent être qu'approchantes. Ainsi, les maladies d'origine tellurique sont dominées par les irrégularités du climat. Les enzooties de peste bovine subissent des poussées cycliques selon un rythme régulier. Après le passage d'une enzootie grave, il faut un délai permettant que les descendants réceptifs soient assez nombreux pour reconstituer un matériel inflammable.

Les chiffres des pertes dues aux contagions sont encore très élevés et justifient un perfectionnement des moyens de défense, notamment contre la peste bovine. Tout progrès dans ce sens se traduira par un accroissement du cheptel et du nombre de bétail que le Cambodge pourra exporter. A l'inverse, une régression dans la mise en cuvre du programme de défense entraînerait des hécatombes, une lente reconstitution du cheptel et l'arrêt progressif des exportations de bétail.

L'utilisation du vaccin pulpe contre la peste bovine date de 1931. Les recensements n'indiquent guère l'action bénifique de ce procédé vaccinal sur l'accroissement du cheptel. Par contre, le flux de l'exportation qui, depuis 194l, a atteint un débit élevé et constant, reflète l'efficacité dè la nouvelle technique.

Les bénéfices que le pays peut retirer de l'exportation sont incomparablement plus élevés que les dépenses de la vaccination antipestique, 'sans laquelle toute production excédentaire de bétail serait impossible. Devant le profit de l'opération, les pouvoirs publics seront de mieux en mieux disposés à favoriser la défense antipestique par vaccination, selon une ampleur qu'elle n'a jamais atteinte et prendront à la charge de la collectivité les dépenses considérables que représentera la consommation de vaccin. 